CSF, myelography demonstrating serpiginous filling defects and increased spinal cord diameter, and a spinal angiographic outline of the AVM with a large intraspinal aneurysmal sac. Following embolizations, clipping of feeding vessels, and surgical removal of the sac, the AVM was closed but the paraplegia had persisted at $10 \mathrm{mo}$ follow-up. (Esparza J et al. Arteriovenous malformation of the spinal cord in the neonate. Child's Nerv Syst 1987; $\underline{3}(5)$ : 301-303).

COMMENT. Spinal cord A-V malformations may be dorsal extradural, compact intraspinal or diffuse intraspinal involving several vertebral segments. The latter presents in childhood or adolescence and carries a poor prognosis. Stereotaxic radiosurgery or proton beam therapy may offer better results than surgical intervention for large AVM's involving eloquent nervous tissue. (Kjellberg RN et al. N Engl J Med 1983;309:269.

\title{
CEREBRAL A-V MALFORMATION IN NEONATE
}

A baby girl who developed congestive heart failure at 3 days of age and was shown to have an aneurysm of the vein of Galen is reported from the Dept Child Health, The Queen's Univ Belfast, Royal Maternity Hosp and Dept Radiology, Royal Victoria Hosp, Belfast. Treatment by embolization with helical stainless steel coils inserted along the straight sinus occluded the aneurysm. Postoperatively, recovery was rapid, the cranial bruit disappeared, and medical treatment for heart failure was discontinued. At 21 mo follow-up, the heart and head circumferences, and growth and development were normal. (McCord FB, Shield MD et al. Cerebral arteriovenous malformation in a neonate: treatment by embolization. Arch Dis Child Dec 1987; 62 (12):1273-1275.

COMENT. In this case non-surgical treatment was successful. Ischemic brain lesions resulting from a steal phenomenon directing blood toward the aneurysm, as reported by Norman and Becker ( $\underline{J}$ Neurol Neurosurg Psychiat $1974 ; 37: 252$ ), did not result.

\section{PAPILLEDIMA IN CHILDREN}

The use of oral fluorescein in the diagnosis of early papilledema in 23 children aged 1 mo to 10 yrs is reported from the Dr Rajendra Prasad Centre for Ophthalmic Sciences, All-India Inst of Med Sciences, Ansari Nagar, New Delhi, India. Of 15 children with suspected or early papilledema associated with hydrocephalus (10), seizures (3), possible tumor (1), and unilateral proptosis (1), late disc staining and retinal vascular fluorescence occurred in 12, the fluorescence at $60 \mathrm{~min}$ being significantly greater or of equal intensity to that at $30 \mathrm{~min}$, denoting a positive test. All cases positive on oral fluorescein showed CT evidence of raised intracranial pressure, while those with negative fluorescein tests had normal CT's. In 8 children with pseudopapilledema examined after oral fluorescein, the retinal vascular fluorescence and slight disc head staining with sharp margins at $30 \mathrm{~min}$ declined markedly by $60 \mathrm{~min}$, a negative result, identical to that found in normal fundi. The authors caution that a negative result may occur with very early stages of papilledema manifested only by venous engorgement. (Ghose S, Nayak BK. Role of oral fluorescein in the diagnosis of early papilledema in children. Brit J Ophthalmol Dec 1987; 71 (12):910-915).

COMMENT. The necessity for conventional intravenous administration of fluorescein of ten precludes its use in small children with suspected papilledema. Oral fluorescein offers a more practical test that may gain acceptance if these results are confirmed. The funduscopic examination and 
diagnosis of early papilledema is often difficult, especially in small children, even for the experienced pediatric neurologist. Ophthalmologists may negate the neurologist's suspicions, but CT scan is nonetheless advisable if the clinical picture suggests a space-occupying lesion.

\section{CONGENITAL MALFORMATIONS}

\section{NEDRAL TUBE DEFECTS}

In an overview of neural tube defects (NID's), Dr RJ Lemire of the Dept Pediatrics, Univ of Washington and Children's Hospital, Seattle, WA, divides them into two major groups: (1) neurulation and (2) postneurulation defects.

Neurulation defects arising between the 17th and 30th day after fertilization are caused by nonclosure of the neural tube, leaving nervous tissue exposed, whereas postneurulation NID's are covered by skin. Three general categories of neurulation defects are described: (1) craniorachischis (total dysraphism), (2) anencephaly, and (3) meningomyelocele. Environmental teratogenic factors implicated in neurulation defects include valproate sodium and nutritional and vitamin deficiencies (see Ped Neur Briefs 1987;1:15). Prenatal diagnosis is made by screening for maternal serum a-fetoprotein (AFP) levels during the 16th-18th week of pregnancy with follow-up ultrasound and amniocentesis when AFP is elevated. Elevated amniotic fluid acetylcholinesterase levels are confirmatory of open NID and eliminate possible false-positive results of AFP tests. The population incidence of open NID's is about $2 / 1000$ births but the chance of recurrence is $1 / 20$.

Postneurulation or closed NID's arising after the 30th day of fetal life include hydrocephalus, encephalocele, and Iumbosacral lesions. The causes of hydrocephalus and associated abnormalities are listed as follows: ArnoldChiari malformation with meningomyelocele, tumors and cysts, aqueductal stenosis, achondroplasia, tuberous sclerosis, Dandy-Walker syndrome, chromosome trisomy 13 and 18 anomalies, prenatal infection, and aneurysm of the vein of Galen. Comprehensive lists of lumbosacral NID's and encephalocele syndromes are provided. Early resection of caudal NID's is advised when practical. (Lemire RJ. Neural tube defects. JAMA Jan 22/29 1988; 259:558-562).

COMENT. As an encouraging postscript to this depressing subject, the author notes a declining incidence of NID's in several areas of the world, including the U.S., related in part to prenatal diagnosis, genetic counselling and nutritional supplementation. Folate treatment before and at the time of conception prevent recurrence of spina bifida. Exposure to spermicide contraceptives is not a risk factor. (See Ped Neur Briefs Aug 1987; $1(3): 15)$. A late occurring intrauterine cause of hydrocephalus is reported in the following paper.

\section{CONGENITAL HYDROCEPHALUS}

Intrauterine intraventricular hemorrhage occurring about 2 weeks or more prior to birth was the cause of congenital hydrocephalus in 4 newborn infants reported from the Abteilung Neonatologie, Universitats-Kinderklinik, Rumelinstrasse 23; D-7400 Tubingen, FR Germany. Multiple pregnancy was an associated risk factor in 2 cases and a hemorrhagic diathesis was present or suspected in 2. Intrauterine diagnosis of subependynal/intraventricular hemorrhage may be made by sonography of the fetal brain when indicated, especially in multiple pregnancy, hemorrhagic diathesis by history, fetal growth retardation, and signs of distress. Postnatally, cerebral ultrasound, CT and examination of the CSF for siderophages may be confirmatory. (Leidig E et al. Intrauterine development of posthemorrhagic hydrocephalus. Eur J Pediat Jan 1988; 147: 26-29). 